\title{
Relationship between Blood Mercury Concentration and Bone Mineral Density in Korean Men in the 2008-2010 Korean National Health and Nutrition Examination Survey
}

\author{
Yang Hee Kim, Jae Yong Shim*, Min Seok Seo, Hyung Ji Yim, Mi Ra Cho \\ Department of Family Medicine, Gangnam Severance Hospital, Yonsei University College of Medicine, Seoul, Korea
}

Background: The results of previous studies on the association between blood mercury $(\mathrm{Hg})$ and bone mineral density (BMD) are inconsistent. We therefore used a large-scale nationwide representative sample of Korean men to investigate the relationship between these two parameters.

Methods: A nationwide cross-sectional study was conducted using data from the 2008 to 2010 Korean National Health and Nutrition Examination Survey to evaluate the relationship between blood Hg and BMD and the prevalence of osteopenia or osteoporosis in 1,190 men over 50 years of age. BMD was measured by dual-energy X-ray absorptiometry. Osteopenia and osteoporosis were diagnosed for each body site according to World Health Organization T-score criteria.

Results: After adjusting for age, body mass index, caloric energy and calcium intake, vitamin D levels, fish consumption, alcohol consumption, smoking, and exercise, quartiles of blood Hg were positively associated with femur neck T-scores in multiple linear regression analysis $(\beta=0.06, \mathrm{P}$-value $=0.03)$. Compared with the lowest blood $\mathrm{Hg}$ quartile, the odds ratios and $95 \%$ confidence intervals for diagnosis of osteopenia or osteoporosis in the second and fourth quartiles were 0.63 (0.41-0.99) and 0.57 (0.36-0.91), respectively, in the femur neck after adjusting for the same co-variables.

Conclusion: High blood Hg levels were associated with reduced odds of decreased femur neck BMD in Korean men. However, subgroup analysis did not show a significant protective effect of blood $\mathrm{Hg}$ on osteoporotic fractures. Further research is necessary to clarify the association between blood Hg and BMD.

Keywords: Mercury; Bone Density; Osteoporosis; Osteoporotic Fractures; Men 


\section{INTRODUCTION}

Osteoporosis is increasingly recognized as an important public health issue in men. ${ }^{1)}$ According to national health survey data from 2008 to 2011, the prevalence of osteoporosis and osteopenia in Korean men older than 50 years of age was $7.3 \%$ and $46.5 \%$, respectively. ${ }^{2)}$ Osteoporosis is a 'silent disease,' typically progressing without symptoms until a fracture occurs. ${ }^{3)}$ Since fractures associated with osteoporosis tend to occur at older ages in men than in women, morbidity and mortality are high in men compared with women. In this regard, early detection and treatment of men with osteoporosis is important, particularly due to increasing life expectancies. ${ }^{1)}$ However, osteoporosis remains largely underdiagnosed, mainly due to the view of osteoporosis solely as a 'women's disease' and the consequent infrequency of bone mineral density (BMD) screening in men., ${ }^{1,3,4)}$

Mercury (Hg) is a toxic heavy metal; individuals are mainly exposed by occupational inhalation of Hg vapor, dental amalgam, and consumption of contaminated fish and shellfish. ${ }^{5,6)}$ Methylmercury, the predominant form of organic $\mathrm{Hg}$, accumulates up the food chain and becomes the main food source of $\mathrm{Hg}$ exposure in humans. ${ }^{7)} \mathrm{Blood} \mathrm{Hg}$ concentration is considered the most valid biomarker of recent methylmercury exposure. ${ }^{8)}$ Due to growing societal concerns about environmental heavy metals in Korea, heavy metal biomonitoring has been performed since the third Korean National Health and Nutritional Examination Survey (KNHANES). ${ }^{9)}$

Previous studies have reported the detrimental effects of heavy metals such as lead and cadmium on bone health. ${ }^{10,11)}$ However, previous reports have showed contrasting results for Hg compared to other heavy metals, leaving unresolved questions regarding the mechanism of the reported protective association between $\mathrm{Hg}$ level and BMD. Several cross-sectional studies reported that serum $\mathrm{Hg}$ levels seemed to have protective effects on bone. ${ }^{12,13)}$ In contrast, experimental studies have reported the opposite. ${ }^{14)}$

Our study evaluated the relationship between blood Hg concentration and BMD in a representative sample of Korean men, who are less influenced by physiological hormonal changes than women. We also analyzed the association between blood $\mathrm{Hg}$ concentration and past incidence of fracture.

\section{METHODS}

\section{Study Population}

This study was based on data obtained from the KNHANES, performed by the Korean Ministry of Health and Welfare from 2008 to 2010, specifically from the KNHANES IV (2008 and 2009 data) and V (2010 data). This complex, stratified, multistage, probability-cluster survey was a nationwide representative study in South Korea that consisted of the Health, Health Examination, and Nutrition Surveys. Among 29,235 participants enrolled in the KNHANES study from 2008 to 2010 , data from men older than 50 years of age, including BMD results, were used. Of these 4,384 subjects, 3,193 were excluded for miss- ing blood Hg laboratory data (participants were randomly selected for heavy metal measurement). We also excluded one participant with outlier Hg levels (168.49 mcg/L). A total of 1,190 Korean men 50-87 years of age were included in the final analysis.

\section{Data Collection}

Information on age and health behaviors including smoking history, alcohol intake, and exercise was collected during health interview surveys. Health behaviors were assessed by questioning participants' habits during the month prior to the interview. Smoking history was divided in two categories: never smoker and former (ex-smoker of $>100$ cigarettes)/current smoker. Alcohol intake was divided in two categories: non- or current drinker. Non-drinkers included men who had never consumed alcohol, those who had not drunk in the previous year, and those who usually drank less that once a month. Exercise was evaluated using the International Physical Activity Questionnaire and categorized into three groups according to the number of days that weight-bearing exercise, such as push-ups, sit-ups, dumbbell, barbell, and chin-up bar, were performed in the week prior to the interview (none, 1-2 times/wk, or 3-5 times/wk).

Body mass index (BMI) was calculated as the weight (kilograms) per square of the height (meters). Serum levels of vitamin D were measured using enzymatic methods with a 147 WIZARD gamma counter (PerkinElmer, Turku, Finland). Total caloric energy and daily calcium intake, identified as possible factors related to BMD in previous studies, were calculated based on food items consumed by participants during a 24-hour period, using the 24-hour dietary recall method. Information on fish consumption, a major dietary source of Hg exposure, was also obtained from the nutrition survey, which provides data on the consumption frequency of nine different fish/shellfish-related food items that are most frequently consumed in Korea (mackerel, tuna, yellow croaker, pollock, anchovy, squid, clam, and pickled seafood). The overall consumption frequency was categorized into two groups based on the intake of at least one type of fish or shellfish in the nutrition survey checklist: less than twice per week and more than twice weekly. ${ }^{15)}$ Participants were also asked if they had been previously diagnosed with spine, hip, or wrist fracture by a doctor.

\section{Blood Mercury Measurement}

To assess blood Hg concentration, 3-mL blood samples were collected into ethylenediaminetetraacetic acid tubes during the health examination, and $5.0 \mathrm{~mL}$ nitric acid $\left(\mathrm{HNO}_{3}\right.$, reagent) was added to lyse the samples. Blood Hg levels were measured using the gold-amalgamation method with an Automatic Mercury Analyzer (DMA-80; Milestone, Bergamo, Italy). The limit of detection for $\mathrm{Hg}$ was $0.158 \mathrm{mg} / \mathrm{L}$. Sample analyses were carried out by Neodin Medical Institute, a laboratory certified by the Korean Ministry of Health and Welfare. The internal quality assurance for Hg measurement was achieved by obtaining standard reference material, and the external quality assurance was commissioned by the German Environmental Quality Assessment Scheme. 


\section{Bone Mineral Density Measurement}

BMD (grams per square centimeter) was measured by dual-energy Xray absorptiometry (DISCOVERY-W, QDR -4500A; Hologic, Bedford, MA, USA) at central skeletal sites (total hip, femur neck, and lumbar spine [L1-L4]). The precision error was $1.8 \%$ for total hip BMD, $2.5 \%$ for femur neck BMD, and $1.9 \%$ for lumbar spine BMD.

BMD was converted into T-scores, a deviation value from the BMD weight-adjusted average peak of a race- and sex-matched Asian (Japanese) healthy population. Participants were classified into three groups according to the T-scores of each site (normal BMD: T-score $\geq-1$, osteopenia: $-2.5 \leq \mathrm{T}$-score $<-1$, osteoporosis: T-score $\leq-2.5$ ).

\section{Statistical Analysis}

Data were expressed as means \pm standard error for continuous variables and numbers and percentages (\%) for categorical variables. Blood $\mathrm{Hg}$ levels were categorized according the quartile of distribution (Q1 33.564, Q2=3.564-5.441, Q3=5.441-8.002, Q4>8.002). To analyze baseline characteristics according to blood $\mathrm{Hg}$ quartiles, we used oneway analysis of variance and chi-square tests for continuous variables and categorical variables, respectively. In order to compare total hip, femur neck, and lumbar spine T-scores according to blood Hg quartiles, multiple stepwise linear regression analysis was performed using T-score as a dependent variable and blood Hg concentrations as independent variables and age, BMI, intake of caloric energy and calcium, vitamin D level, fish consumption, alcohol intake, cigarette smoking, and exercise as covariates. Statistical analysis was performed using
IBM SPSS Statistics for Windows ver. 20.0 (IBM Co., Armonk, NY, USA). All statistical tests were two-sided, and P-values less than 0.05 were considered statistically significant.

\section{RESULTS}

Osteopenia and osteoporosis were present in $48.4 \%$ and $5.5 \%$ of the study participants, respectively. Table 1 shows their basic characteristics according to blood Hg quartiles. Men with higher blood Hg levels were more likely to be younger and current alcohol drinkers with higher BMI, total energy and calcium intake, and fish consumption. The mean T-scores of the whole femur and femur neck were significantly higher according to the quartiles of blood $\mathrm{Hg}$ (P-value $<0.001)$.

Stepwise multiple linear regression analysis using the T-score of each BMD site and blood Hg quartile as the dependent and independent variables, respectively, revealed that whole femur and femur neck BMDs were positively associated with blood $\mathrm{Hg}$ levels after adjusting for age, BMI, alcohol intake, cigarette smoking, and exercise (model 1: $\beta=0.06$ and 0.08 , P-values $=0.02$ and 0.001 , respectively) (Table 2). After further adjusting for intake of caloric energy and calcium, fish consumption, and serum vitamin D levels, the relationship between femur neck BMD and blood Hg level quartiles remained significant (model 2: $\beta=0.06$, P-value=0.03) (Table 2).

Moreover, the association between $\mathrm{Hg}$ level quartiles and prevalence of osteopenia and osteoporosis in the femur neck remained statistically significant in multivariate logistic regression analysis after ad-

Table 1. Basic study participant characteristics, according to quartiles of blood Hg level

\begin{tabular}{|c|c|c|c|c|c|c|}
\hline \multirow{2}{*}{ Characteristic } & \multicolumn{6}{|c|}{ Blood mercury quartiles (mcg/L) } \\
\hline & Total & Q1 ( $\leq 3.346)$ & Q2 (3.346-5.321) & Q3 (5.321-8.003) & Q4 ( $\geq 8.003)$ & P-value* \\
\hline No. of participant & $1,190(100)$ & $298(25)$ & $297(25)$ & $298(25)$ & $297(25)$ & \\
\hline $\mathrm{Hg}(\mathrm{mcg} / \mathrm{L})$ & $6.581 \pm 0.233$ & $2.583 \pm 0.038$ & $4.505 \pm 0.030$ & $6.567 \pm 0.042$ & $12.676 \pm 0.324$ & \\
\hline Age (y) & $61.09 \pm 0.233$ & $64.11 \pm 0.494$ & $61.49 \pm 0.472$ & $59.55 \pm 0.428$ & $59.20 \pm 0.416$ & $<0.001$ \\
\hline Body mass index $\left(\mathrm{kg} / \mathrm{m}^{2}\right)$ & $23.88 \pm 0.084$ & $23.09 \pm 0.176$ & $23.76 \pm 0.437$ & $24.01 \pm 0.163$ & $24.64 \pm 0.167$ & $<0.001$ \\
\hline Energy intake (kcal/d) & $2,079.75 \pm 24.521$ & $1,887.33 \pm 41.736$ & $2,108.24 \pm 52.503$ & $2,121.93 \pm 47.674$ & $2,204.47 \pm 51.656$ & $<0.001$ \\
\hline Calcium intake (mg/d) & $554.33 \pm 11.957$ & $472.85 \pm 18.621$ & $536.59 \pm 26.219$ & $623.71 \pm 26.080$ & $587.34 \pm 22.676$ & 0.004 \\
\hline Smoking status & & & & & & 0.84 \\
\hline Never smoker & $759(63.8)$ & $193(65.0)$ & $193(64.8)$ & $187(62.8)$ & $186(62.6)$ & \\
\hline Former/current smoker & $421(35.4)$ & $102(34.3)$ & $102(34.2)$ & $107(35.9)$ & $110(37.0)$ & \\
\hline Drinking status & & & & & & 0.04 \\
\hline Non-drinker & $359(30.2)$ & $125(42.1)$ & $84(28.2)$ & $76(25.5)$ & $74(24.9)$ & \\
\hline Current drinker & $819(68.8)$ & $170(57.1)$ & $210(70.5)$ & $218(73.2)$ & $221(74.4)$ & \\
\hline Weight-bearing exercise & & & & & & 0.91 \\
\hline None & $756(63.5)$ & $196(66.0)$ & $190(63.8)$ & $179(60.1)$ & $191(64.3)$ & \\
\hline $1-2$ times/wk & $141(11.8)$ & $34(11.4)$ & $33(11.1)$ & $35(11.7)$ & $39(13.1)$ & \\
\hline 3-5 times/wk & $284(23.9)$ & $65(21.9)$ & $72(24.2)$ & $81(27.2)$ & $66(22.2)$ & \\
\hline Fish consumption & & & & & & 0.005 \\
\hline Less than twice a week & $399(33.5)$ & $119(41.2)$ & $95(32.0)$ & $76(25.5)$ & $109(36.7)$ & \\
\hline More than twice a week & $614(51.6)$ & $141(47.3)$ & $153(51.5)$ & $170(57.0)$ & $150(50.5)$ & \\
\hline Whole body bone mineral density $\left(\mathrm{g} / \mathrm{cm}^{2}\right)$ & $1.160 \pm 0.004$ & $1.144 \pm 0.008$ & $1.177 \pm 0.008$ & $1.162 \pm 0.007$ & $1.178 \pm 0.007$ & 0.04 \\
\hline Osteopenia and osteoporosis & $515(43.3)$ & $162(54.4)$ & $115(38.7)$ & $133(44.6)$ & $105(35.4)$ & 0.05 \\
\hline
\end{tabular}

Values are presented as number (\%) or mean \pm standard error. $\mathrm{Hg}$, mercury.

${ }^{*}$ Calculated using 1 -way analysis of variance test or $\chi^{2}$ test. 
justing for the same potential confounders as model 2 (Table 3). Men with blood Hg levels in the second and fourth quartiles had decreased odds of reduced femur neck BMD (adjusted odds ratio [OR], 0.62; 95\% confidence interval [CI], 0.40 to 0.96 for Q2; adjusted OR, 0.57; $95 \% \mathrm{CI}$, 0.36 to 0.91 for Q4). In the case of total hip, men with blood $\mathrm{Hg}$ concentrations in the fourth quartile had 0.36 - and 0.47 -fold decreased risks of having osteopenia and osteoporosis in models 1 and 2, respectively, compared with men with levels in the first quartile (reference group).

Subgroup analysis was used to assess the association between past fracture and blood Hg quartile as dependent and independent variables, respectively, using the KNHANES V survey data from 2010 (the only year for which fracture history was available). Regardless of covariates, there was no significant correlation between the two parameters. Interestingly, in this multiple regression analysis, the ORs history of fracture in the second and fourth quartiles of blood $\mathrm{Hg}$ consistently showed a tendency to decrease compared to the first quartile (Table 4).

Table 2. Multiple linear regression analysis of T-scores for different bone mineral density, sites according to quartiles of blood mercury level

\begin{tabular}{|c|c|c|c|c|c|c|}
\hline & \multicolumn{3}{|c|}{ Model $1^{*}$} & \multicolumn{3}{|c|}{ Model $2^{\dagger}$} \\
\hline & $\beta$ & $\operatorname{SE}(\beta)$ & P-value & $\beta$ & SE $(\beta)$ & P-value \\
\hline Total hip & 0.06 & 0.07 & 0.02 & 0.02 & 0.03 & 0.43 \\
\hline Femur neck & 0.08 & 0.09 & 0.001 & 0.06 & 0.07 & 0.03 \\
\hline Lumbar spine & -0.02 & -0.01 & 0.65 & -0.04 & -0.04 & 0.31 \\
\hline
\end{tabular}

\section{SE, standard error.}

*Model 1 was adjusted for age, body mass index, alcohol intake, cigarette smoking, and exercise. ${ }^{\dagger}$ Model 2 was further adjusted for intake of caloric energy and calcium, fish consumption, and vitamin D level in addition to the corrections included in model 1.

Table 3. OR $(95 \% \mathrm{Cl})$ for prevalence of osteopenia and osteoporosis according to quartiles of blood mercury level

\begin{tabular}{cccc}
\hline & Osteopenia/osteoporosis & Model $1^{*}$ & ${\text { Model } 2^{\dagger}}$ \\
\hline Total hip & & & 1 \\
Q1 & $59(22.52)$ & 1 & $0.76(0.40-1.42)$ \\
Q2 & $29(12.03)$ & $0.72(0.42-1.23)$ & $0.55(0.27-1.12)$ \\
Q3 & $24(10.62)$ & $0.57(0.32-1.00)$ & $0.47(0.21-0.98)$ \\
Q4 & $13(5.56)$ & $0.36(0.18-0.71)$ & 1 \\
Femur neck & & 1 & $0.63(0.41-0.99)$ \\
Q1 & $144(54.96)$ & $0.60(0.40-0.89)$ & $0.79(0.51-1.24)$ \\
Q2 & $86(35.68)$ & $0.80(0.54-1.20)$ & $0.57(0.36-0.91)$ \\
Q3 & $96(38.40)$ & $0.54(0.36-0.83)$ & 1 \\
Q4 & $69(29.49)$ & & $0.85(0.55-1.31)$ \\
Lumbar spine & & 1 & $1.09(0.70-1.68)$ \\
Q1 & $120(46.88)$ & $0.75(0.51-1.11)$ & $0.91(0.59-1.43)$ \\
Q2 & $83(36.24)$ & $1.02(0.69-1.50)$ & $0.85(0.57-1.26)$ \\
Q3 & $102(41.80)$ & & \\
Q4 & $83(35.78)$ & & \\
\hline
\end{tabular}

Values are presented as number (\%) or OR $(95 \% \mathrm{Cl})$.

$\mathrm{OR}$, odds ratio; $\mathrm{Cl}$, confidence interval.

${ }^{*}$ Model 1 was adjusted for age, body mass index, alcohol intake, cigarette smoking, and exercise. ${ }^{\dagger}$ Model 2 was further adjusted for intake of caloric energy and calcium, fish consumption, and vitamin D level in addition to the corrections included in model 1.

Table 4. Odds ratio ( $95 \% \mathrm{Cl})$ for history of fracture, according to blood $\mathrm{Hg}$ quartile $(\mathrm{mcg} / \mathrm{L})$

\begin{tabular}{ccccc}
\hline & Past history of fracture & Age-adjusted & Fish consumption-adjusted & Multivariable*-adjusted \\
\hline Blood Hg quartiles & & & & 1 \\
Q1 $(\leq 3.347)$ & $12(12.5)$ & 1 & $0.99(0.38-2.59)$ & $0.81(0.27-2.42)$ \\
Q2 (3.347-5.337) & $12(12.90)$ & $0.96(0.40-2.27)$ & $1.28(0.52-3.15)$ & $1.08(0.39-3.01)$ \\
Q3 (5.337-8.014) & $16(17.02)$ & $1.26(0.55-2.86)$ & $0.68(0.24-1.87)$ & $0.61(0.2-1.83)$ \\
Q4 (>8.014) & $10(10.64)$ & $0.73(0.29-1.80)$ & \\
\hline
\end{tabular}

Values are presented as number $(\%)$ or $\mathrm{OR}(95 \% \mathrm{Cl})$.

$\mathrm{OR}$, odds ratio; $\mathrm{Cl}$, confidence interval; $\mathrm{Hg}$, mercury.

${ }^{*}$ Adjustments for age, body mass index, alcohol intake, cigarette smoking, exercise, intake of caloric energy and calcium, fish consumption, and vitamin D level, and whole body bone mineral density. 


\section{DISCUSSION}

We investigated the association between blood Hg level and BMD in Korean men, a population with an increasing prevalence of osteoporosis and related fractures. Increased blood Hg levels were significantly associated with increased total hip and femoral neck T-scores, as well as decreased prevalence of osteopenia and osteoporosis at the same skeletal sites. No associations were found between blood $\mathrm{Hg}$ level and lumbar spine BMD. In addition, the fracture incidence seemed to decrease as blood Hg concentration increased, although this observation was not statistically significant.

Studies on the effects of $\mathrm{Hg}$ exposure in bones relatively scarce, and further research is necessary to clarify the mechanism. Methylmercury, a well-known neurotoxicant, unexpectedly showed positive correlation with BMD in previous cross-sectional studies. Cho et al. ${ }^{12)}$ reported a significantly decreased risk of osteoporosis among postmenopausal women with higher Hg levels. Similarly, Pollack et al. ${ }^{13)}$ suggested a negative association between $\mathrm{Hg}$ and odds of decreased lumbar spine BMD in healthy, premenopausal women. As boys are more susceptible to neurotoxic effects of methylmercury than girls because $\mathrm{Hg}$ is easily transferred to the fetus of pregnant woman, we suspected a possible gender difference in BMD changes in adults after Hg exposure. ${ }^{16)}$ Although the positive correlation between $\mathrm{Hg}$ concentration and BMD was consistent for men and women, the BMD site of significant correlation differed between sexes. While Hg influenced the lumbar spine of women in this study, Hg exposure significantly affected the femur neck in men. This difference may be explained by the perimenopausal loss of bone mass most pronouncedly in the lumbar spine of women compared to a small longitudinal bone loss in the hip throughout life in men. ${ }^{17)}$ Also, while both Hg level and hip BMD decreased with advancing age, spine BMD increased with aging, similar to results of a previous study. ${ }^{18)}$ This gender discrepancy might be partly due to gender differences in serum Hg levels. Considering that the femoral neck has a higher proportion of cortical bone than the lumbar spine, cortical bone could be more affected by Hg level. However, this issue requires further investigation.

Blood Hg levels are used to detect relatively recent exposure to methylmercury. A commonly used reference interval for blood $\mathrm{Hg}$ is 0.6-59.0 mcg/L, and blood Hg levels higher than $10 \mathrm{mcg} / \mathrm{L}$ generally indicates an unusual level for someone who does not regularly work with Hg. Methylmercury is distributed to other body tissue, like the brain and kidneys, which lowers blood Hg levels. ${ }^{5)}$ In our study, Hg concentration showed a normal distribution, with a mean value of $6.581 \mathrm{mcg} / \mathrm{L}$; the $\mathrm{Hg}$ quartile ranges in this study were $0.683-3.564$ $\mathrm{mcg} / \mathrm{L}, 5.565-5.411 \mathrm{mcg} / \mathrm{L}, 5.417-8.002 \mathrm{mcg} / \mathrm{L}$, and 8.003-42.753 $\mathrm{mgc} / \mathrm{L}$. Therefore, we must interpret our results within the normal range of blood $\mathrm{Hg}$ concentrations, and emphasize that our findings do not imply that the benefit increases with mercury exposure. Further research on the ranges of $\mathrm{Hg}$ levels that result in beneficial and toxic effects is required.

Yachiguchi et al. ${ }^{19)}$ suggested that short-term Hg exposure may be involved in the protection of osteoblasts, based on the increased expression of metallothionein, a metal-binding protein of osteoblasts, in marine teleost scales used as a model system of bone. However, Suzuki et al. ${ }^{14)}$ evaluated the effects of $\mathrm{Hg}$ on bone metabolism in scale bone cells and reported that $\mathrm{Hg}$ exposure affects calcium homeostasis, resulting in osteoclast activation and inhibition with acute and longtime exposure, respectively. It is also well known that estrogen deficiency causes osteoporosis in postmenopausal women and contributes to the development of osteoporosis in elderly men. Associated estrogen-dependent cytokine changes inhibit bone resorption. ${ }^{20)}$ In an experimental study, Wang et al. ${ }^{21)}$ reported that mercury chloride showed estrogen-like effects through binding and activation of the estrogen receptor in rat uteri. In contrast, Davis et al. ${ }^{22)}$ reported that inhalation of $\mathrm{Hg}$ vapor altered estrous cyclicity in female rats, indicating possible reproductive toxicity. Therefore, further studies on the physiology of bone mineralization and $\mathrm{Hg}$ are required to support our findings.

This study has several limitations. First, as with all cross-sectional studies, it is difficult to determine the causal pathways of the observed relationships between blood Hg concentration and BMD level. Moreover, with a half-life of three to five days, $\mathrm{Hg}$ is either excreted or accumulated in solid organs; therefore, blood Hg levels only reflect recent exposure and do not correlate with total body burden. ${ }^{23)}$ Because osteoporosis develops over a longer time period, blood $\mathrm{Hg}$ level is a limited biomarker for identifying associations. ${ }^{12)}$ Recently, 2,3 dimercapto1-propanesulfonate, a chelator of $\mathrm{Hg}$, has been recognized as a reliable estimate of body burden by a number of investigators. ${ }^{5)}$ Further research using this chelator may be helpful in the future. Third, sources of inorganic Hg, typically inhalation of vaporized gas in industrial settings and dental amalgams, were not considered in this study. ${ }^{9)}$ While previous studies have reported fish consumption to be the primary source of $\mathrm{Hg}$ in Korea, You et al. ${ }^{7)}$ reported that dietary Hg intake was relatively weakly correlated with blood $\mathrm{Hg}$ concentrations. According to Kim and Lee, ${ }^{24)}$ no significant association between blood $\mathrm{Hg}$ and dental amalgam treatment was observed in a study based on KNHANES data. This implies that Hg may have an effect via pathophysiological pathways other than those related to fish consumption. Also, the numbers of participants who were classified as osteoporosis for each BMD site were too small to assess the ORs for osteoporosis separately from osteopenia. Thus, a larger study group is warranted in the future. Finally, the association between blood mercury concentration and fracture risk may have been insignificant since we used data of history of fracture. Further prospective population studies are necessary to clarify whether high Hg levels are associated with both higher mineral density and lower risk of fracture.

In conclusion, our analysis of nationwide survey data showed that high blood Hg levels may be positively associated with increased total hip and femur neck BMD, but do not offer significant protection against fragility-associated fractures. Further experimental studies and research on the mechanism of the preventive effects of blood $\mathrm{Hg}$ on $\mathrm{BMD}$ are required. 


\section{CONFLICT OF INTEREST}

No potential conflict of interest relevant to this article was reported.

\section{REFERENCES}

1. Kim LO, Kim HJ, Kong MH. A new predictive index for osteoporosis in men under 70 years of age: an index to identify male candidates for osteoporosis screening by bone mineral density. J Osteoporos 2014;2014: 781897.

2. Park EJ, Joo IW, Jang MJ, Kim YT, Oh K, Oh HJ. Prevalence of osteoporosis in the Korean population based on Korea National Health and Nutrition Examination Survey (KNHANES), 2008-2011. Yonsei Med J 2014;55:1049-57.

3. NIH Osteoporosis and Related Bone Diseases National Resource Center. Osteoporosis in men [Internet]. Bethesda (MD): NIH Osteoporosis and Related Bone Diseases National Resource Center; 2015 [cited 2015 Jun 30]. Available from: http://www.bones.nih.gov.

4. Willson T, Nelson SD, Newbold J, Nelson RE, LaFleur J. The clinical epidemiology of male osteoporosis: a review of the recent literature. Clin Epidemiol 2015;7:65-76.

5. Agency for Toxic Substances and Disease Registry. Toxicology profile for mercury [Internet]. Atlanta (GA): Agency for Toxic Substances and Disease Registry; 1999 [cited 2015 Oct 9]. Available from: http://www. atsdr.cdc.gov/toxprofiles/tp.asp?id=115\&tid=24\#.

6. Jarup L. Hazards of heavy metal contamination. Br Med Bull 2003;68: 167-82.

7. You CH, Kim BG, Kim YM, Lee SA, Kim RB, Seo JW, et al. Relationship between dietary mercury intake and blood mercury level in Korea. J Korean Med Sci 2014;29:176-82.

8. Weil M, Bressler J, Parsons P, Bolla K, Glass T, Schwartz B. Blood mercury levels and neurobehavioral function. JAMA 2005;293:1875-82.

9. Lee YJ, Hwang IC. Relationship between serum ferritin level and blood mercury concentration using data from the Korean national health and nutrition examination survey (2010-2012). Environ Res 2014;135: 271-5.

10. Brito JA, Costa IM, E Silva AM, Marques JM, Zagalo CM, Cavaleiro II, et al. Changes in bone $\mathrm{Pb}$ accumulation: cause and effect of altered bone turnover. Bone 2014;64:228-34.

11. Engstrom A, Michaelsson K, Vahter M, Julin B, Wolk A, Akesson A. Associations between dietary cadmium exposure and bone mineral density and risk of osteoporosis and fractures among women. Bone
2012;50:1372-8.

12. Cho GJ, Park HT, Shin JH, Hur JY, Kim SH, Lee KW, et al. The relationship between blood mercury level and osteoporosis in postmenopausal women. Menopause 2012;19:576-81.

13. Pollack AZ, Mumford SL, Wactawski-Wende J, Yeung E, Mendola P, Mattison DR, et al. Bone mineral density and blood metals in premenopausal women. Environ Res 2013;120:76-81.

14. Suzuki N, Yamamoto M, Watanabe K, Kambegawa A, Hattori A. Both mercury and cadmium directly influence calcium homeostasis resulting from the suppression of scale bone cells: the scale is a good model for the evaluation of heavy metals in bone metabolism. J Bone Miner Metab 2004;22:439-46.

15. Bolam KA, van Uffelen JG, Taaffe DR. The effect of physical exercise on bone density in middle-aged and older men: a systematic review. Osteoporos Int 2013;24:2749-62.

16. Vahter M, Akesson A, Liden C, Ceccatelli S, Berglund M. Gender differences in the disposition and toxicity of metals. Environ Res 2007;104:85-95.

17. Warming L, Hassager C, Christiansen C. Changes in bone mineral density with age in men and women: a longitudinal study. Osteoporos Int 2002;13:105-12.

18. Lee KM, Kwon SS, Chung CY, Lee SY, Kim TG, Choi Y, et al. Genderand body-site-specific factors associated with bone mineral density in a non-institutionalized Korean population aged $\geq 50$ years. J Bone Miner Metab 2015;33:401-9.

19. Yachiguchi K, Sekiguchi T, Nakano M, Hattori A, Yamamoto M, Kitamura K, et al. Effects of inorganic mercury and methylmercury on osteoclasts and osteoblasts in the scales of the marine teleost as a model system of bone. Zoolog Sci 2014;31:330-7.

20. Riggs BL. The mechanisms of estrogen regulation of bone resorption. J Clin Invest 2000;106:1203-4.

21. Wang YD, Chen XY, Wu YM, Xu D. Experiment study on the estrogenlike effect of compounds of mercury, chromium and manganese. Wei Sheng Yan Jiu 2005;34:49-51.

22. Davis BJ, Price HC, O'Connor RW, Fernando R, Rowland AS, Morgan DL. Mercury vapor and female reproductive toxicity. Toxicol Sci 2001; 59:291-6.

23. Bernhoft RA. Mercury toxicity and treatment: a review of the literature. J Environ Public Health 2012;2012:460508.

24. Kim Y, Lee BK. Association between blood lead and mercury levels and periodontitis in the Korean general population: analysis of the 2008-2009 Korean National Health and Nutrition Examination Survey data. Int Arch Occup Environ Health 2013;86:607-13. 